A Simplified Presentation of Einstein's Unified Field Equations. By Tullio Levi-Civita. Authorized translation by John Dougall. London, Blackie and Sons, $1929.22 \mathrm{pp}$.

It should be stated at the outset that the author of this pamphlet does not merely elucidate the work of Einstein but attacks the problem ab initio. For the specification both of metrical space and of electromagnetic phenomena he uses, (as did Einstein), the sixteen parameters, $\lambda_{i}^{\alpha}, \alpha=1, \cdots, 4$, defining four orthogonal congruences, $i=1, \cdots, 4 .\left(\lambda_{i}^{\alpha}=i h^{\alpha}\right.$ in Einstein's notation.) The ten coefficients $g_{\alpha \beta}$ in the expression for $d s^{2}$ are completely determined by these parameters, but not conversely. For the complete determination of the parameters ten relations are furnished by the usual Einstein gravitational equations; the remaining six relations come from Maxwell's equations in connection with a (postulated) relation between the electromagnetic field tensor and the parameters. The author avoids the use of "covariant derivatives with respect to the quadruplet" by "making use of perfectly familiar methods of the absolute differential calculus." The necessary fundamentals for a comfortable reading of this pamphlet will be found in Chapters 4-6 and 10-11 of The Absolute Differential Calculus by the same author.

\title{
C. A. Sноок
}

Introduzione alla Geometria Algebrica. By Edgardo Ciani. Padova, A. Milani, 1931. x +599 pp. Mimeographed. Price Lire 60.00.

The present volume is a continuation of the author's Metodo delle Coordinate Proiettive Omogenee (2d edition, Torino, 1928). Thus it presupposes a general knowledge of projective coordinates, of determinants and the elements of invariants, and of the processes of the calculus. It is primarily of didactic value; all proofs are given, and no sources or citations to other methods are given. The book has no figures and is not provided with an index.

The first thing considered is the binary biquadratic form, its two invariants, its covariants, the harmonic and equianharmonic forms, and the syzygetic involution. Then follows a very brief discussion of algebraic plane curves, including Plücker's numbers, the hessian, and points of inflexion, and a similar one for surfaces and space curves. Quadratic (Cremona) correspondences between two planes with a brief treatment of the resolution of singularities of plane algebraic curves precedes the more systematic discussion of quartic curves in two and in three dimensions. A fairly full chapter on cubic surfaces is followed by one on selected quartics, particularly the Kummer surface and the Steiner surface. Much of the author's own work is given in the chapter on geometric applications of the theory of groups. Finally, about one-fourth of the volume is devoted to properties of four-space. It includes the representation of points, lines, planes and three-spaces by equations and parametrically, collineations and correlations, then an outline of quadratic varieties and of the cubic variety.

The mechanical preparation of the volume is excellent, and makes it particularly easy to read. As a general introduction to the field, the book accomplishes the purpose in a satisfactory manner, but it would have been much more valuable had a bibliography been added. 